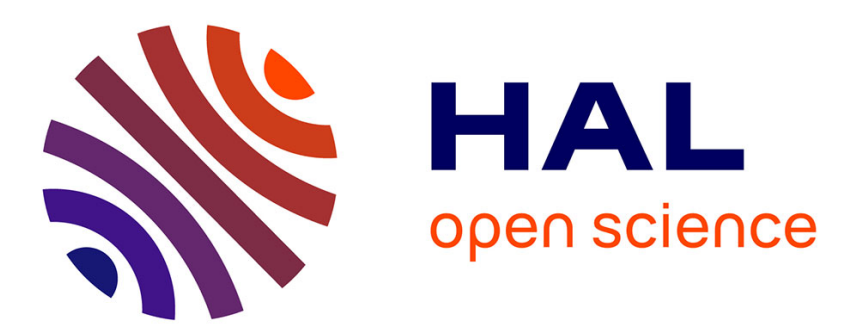

\title{
The red tooth hypothesis: A computational model of predator-prey relations, protean escape behavior and sexual reproduction
}

Robert M. French

\section{- To cite this version:}

Robert M. French. The red tooth hypothesis: A computational model of predator-prey relations, protean escape behavior and sexual reproduction. Journal of Theoretical Biology, 2009, 262 (1), pp.165. 10.1016/j.jtbi.2009.09.016 . hal-00559158

\section{HAL Id: hal-00559158 \\ https://hal.science/hal-00559158}

Submitted on 25 Jan 2011

HAL is a multi-disciplinary open access archive for the deposit and dissemination of scientific research documents, whether they are published or not. The documents may come from teaching and research institutions in France or abroad, or from public or private research centers.
L'archive ouverte pluridisciplinaire HAL, est destinée au dépôt et à la diffusion de documents scientifiques de niveau recherche, publiés ou non, émanant des établissements d'enseignement et de recherche français ou étrangers, des laboratoires publics ou privés. 


\section{Author's Accepted Manuscript}

The red tooth hypothesis: A computational model of predator-prey relations, protean escape behavior and sexual reproduction

Robert M. French

PII: S0022-5193(09)00436-6

DOI: doi:10.1016/j.jtbi.2009.09.016

Reference: YJTBI 5707

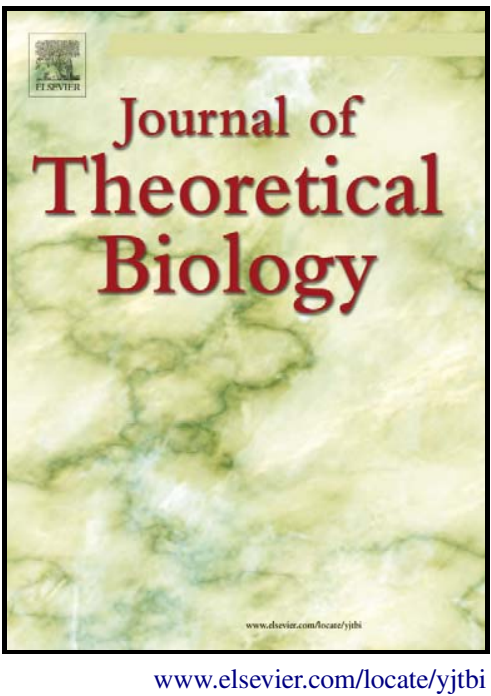

To appear in: $\quad$ Journal of Theoretical Biology

Received date: $\quad 6$ February 2009

Revised date: $\quad 4$ August 2009

Accepted date: 10 September 2009

Cite this article as: Robert M. French, The red tooth hypothesis: A computational model of predator-prey relations, protean escape behavior and sexual reproduction, Journal of Theoretical Biology, doi:10.1016/j.jtbi.2009.09.016

This is a PDF file of an unedited manuscript that has been accepted for publication. As a service to our customers we are providing this early version of the manuscript. The manuscript will undergo copyediting, typesetting, and review of the resulting galley proof before it is published in its final citable form. Please note that during the production process errors may be discovered which could affect the content, and all legal disclaimers that apply to the journal pertain. 
The Red Tooth Hypothesis: A computational model of predatorprey relations, protean escape behavior and sexual reproduction

Robert M. French

LEAD-CNRS, University of Burgundy, Dijon, France

robert.french@u-bourgogne.fr

8 Abstract

9 This paper presents an extension of the Red Queen Hypothesis (hereafter, RQH) that we

10 call the Red Tooth Hypothesis (RTH). This hypothesis suggests that predator-prey

11 relations may play a role in the maintenance of sexual reproduction in many higher

12 animals. RTH is based on an interaction between learning on the part of predators and

13 evolution on the part of prey. We present a simple predator-prey computer simulation that

14 illustrates the effects of this interaction. This simulation suggests that the optimal escape

15 strategy from the prey's standpoint would be to have a small number of highly reflexive,

16 largely innate (and, therefore, very fast) escape patterns, but that would also be

17 unlearnable by the predator. One way to achieve this would be for each individual in the

18 prey population to have a small set of hard-wired escape patterns, but which were

19 different for each individual.

20 We argue that polymorphic escape patterns at the population level could be produced

21 via sexual reproduction at little or no evolutionary cost and would be as, or potentially

22 more, efficient than individual-level protean (i.e., random) escape behavior. We further

23 argue that, especially under high predation pressure, sexual recombination would be a 
1 more rapid, and therefore more effective, means of producing highly variable escape

2 behaviors at the population level than asexual reproduction.

4 Key words: Red Queen Hypothesis, Red Tooth Hypothesis, predation, flight behavior,

5 sex, polymorphic escape sequences, predator-prey, protean escape behavior

\section{Introduction}

9 One of the enduring mysteries of evolutionary biology is the ubiquity of sexual

10 reproduction. If we take as the fundamental postulate of Darwinian evolution that all

11 individuals attempt (unconsciously) to maximize their own genetic material in successive

12 generations, then the cost of sexual reproduction - half of each individual's genetic

13 material - would seem to be enormous. Williams (1975) famously described this as the

14 "cost of meiosis" and claimed that "Nothing remotely approaching an advantage that

15 could balance the cost of meiosis has been suggested." Williams traced the origin of the

16 question to Fisher (1930) and, especially, to Muller (1932), who developed a defense of

17 the advantage of sex based on species selection. Muller argued that recombination

18 through sexual reproduction allowed the immediate introduction into the same lineage of

19 favorable mutations occurring in two different individuals. By contrast, in asexual

20 reproduction, one mutation must occur first in some individual and the second mutation

21 must then occur in a descendant of that same individual, a far unlikelier event than an

22 individual obtaining both favorable mutations through sexual exchange. In this view, not

23 all the genes acquired through sexual reproduction would necessarily be of immediate 
1 benefit to the individual who acquired them. Rather, were the environment to change,

2 these genes would prove adaptive to the individuals possessing them in the new

3 environment, thereby leading to a more rapid evolutionary adaptation of the species. In

4 short, gene recombination achieved through sexual reproduction would reduce the long-

5 term possibility of extinction of the species in an ever-changing environment.

$6 \quad$ However, Crow \& Kimura (1965) and Maynard-Smith (1971) demonstrated

7 (mathematically) that, while gene accumulation through sexual reproduction might result

8 in increased rates of evolutionary adaptation for very large populations, this would not

9 occur for small populations (e.g., for population sizes smaller than 1000, cf. Crow \&

10 Kimura, 1965). We will return to this important point during our discussion of the Red

11 Tooth Hypothesis below.

\section{The Red Queen Hypothesis}

15 Van Valen's (1973) observation that taxonomic survivorship curves (for taxa at or above

16 the level of genera) tended to be log-linear led to a set of new ideas as to why sex and

17 genetic recombination offset the cost of meiosis. He suggested that the observed log-

18 linear species extinction curves were due to co-evolutionary forces among species that

19 were best described as a zero-sum "arms race", the end result of which was no absolute

20 improvement in the average fitness of individuals within a species with respect to the

21 individuals in a competing species. Even though the plausibility of this zero-sum arms

22 race was debated in the literature for a time (e.g., Maynard Smith, 1978; Stenseth \&

23 Maynard Smith, 1984), Van Valen's work set the stage for an explicit statement of the 
1 Red Queen Hypothesis and the suggestion that it was responsible for the existence and

2 maintenance of sexual reproduction in essentially all species of higher animals

3 (Hamilton, 1975, 1980; Levin, 1975; Jaenike, 1978; Bell 1982). Bell (1982, p. 143) wrote

4 “...sex is favoured by interaction with other sexual species because the changing

5 spectrum of genotypes among these other species creates a highly uncertain environment,

6 compels an adaptive genetic response which can be supplied only through

7 recombination." Thus, without recourse to (largely discredited) theories of species

8 selection, RQH specifies the benefits provided by sexual reproduction that would offset

9 its elevated genetic cost to individual organisms. Bell (1982, p.157) named this the Red

10 Queen Hypothesis of sex and recombination, after the Red Queen in Lewis Carroll's

11 Through the Looking Glass who runs as fast as she can to remain in the same place.

12 Jaenike (1978) first introduced the idea of a parasite-based theory of the maintenance

13 of sex and Burt and Bell (1987) refined this idea into what is currently the most common

14 construal of RQH - namely, that "crossing-over [i.e., recombination through sexual

15 reproduction] may function to combat antagonists with short generation times." Burt and

16 Bell's focus was specifically on host-parasite relations and the fact that genetic mixing

17 was essential to allow individuals to successfully combat assaults by parasites. In other

18 words, since the reproductive rates of some parasites are four to five orders of magnitude

19 faster than those of their hosts, for each generation of their host, the parasites have

20 hundreds of thousands of generations of mutation-engendered opportunities to unlock its

21 defense mechanisms. But each individual host is the potential target of many, different

22 parasites and, while a fortunate mutation might provide that individual with an effective

23 defense against one particular parasite, mutation alone would not be sufficient to protect 
1 that individual from the range of parasites likely to attack it. Consequently, RQH posits

2 that only the exchange of genetic material via sexual reproduction would allow some

3 individuals to acquire the full range of protective mechanisms required to fend off a large

4 number of different and ever-changing parasites. So, while the cost of sexual

5 reproduction is half of one's genetic material, at least the half that is sent into the next

6 generation has a better chance of surviving attacks by parasites. In other words, while

7 asexual reproduction would ensure the transmission of all of an individual's genes, the

8 bearers of these unchanging genes would eventually succumb to parasites.

\section{The Red Tooth Hypothesis}

12 The present article fully acknowledges the role of $\mathrm{RQH}$ in the emergence and

13 maintenance of sexual reproduction, but, in addition, suggests that sexual reproduction

14 might also have evolved, and would continue to be maintained, because of predator-prey

15 relations alone, even in the absence of classic parasite-host relations characterized by

16 highly different reproduction rates. (Predation, in this context, will refer to the capture

17 and consumption of one animal by another.) Hereafter, we will refer to this hypothesis as

18 the Red Tooth Hypothesis (RTH), after Tennyson's characterization of nature as being

19 "red in tooth and claw."

21 Assumptions of RTH

23 Four main assumptions underlie RTH. These are: 
22 at the population level, while each individual in the population would have its own small

23 set of optimally rapid, reflexive escape patterns. 
We assume, like Zheng et al. (2005), that a small number of innate elementary

2 escape-movements (EEMs) make up more complex escape patterns and that there are

3 low-level neural correlates for these innate EEMs. There are two basic ways to generate a

4 variety of escape patterns from these innate EEMs at the population level: mutation and

5 recombination. In this paper we will argue that the most efficient way to rapidly generate

6 a wide range of complex escape patterns at the population level - thereby producing what

7 appears to the predator to be protean escape behavior at the individual level (because the

8 predator does not chase the same individual twice) - is by recombination of these EEM

9 genes through sexual reproduction.

10 Clearly, complex, rapid escape patterns made up of sequences of more elementary

11 behaviors are not the only efficient means of escaping from predators that prey have

12 developed. Tortoises, for example, have no need for rapid escape patterns, nor do

13 individual fish in schools, at least until the predator is very close at hand. Some animals

14 never venture far from their refuges and need only to make a straight-line dash for the

15 safety of their refuge whenever a predator threatens. Nevertheless, a great many animals

$16 d o$ flee from predators and remain in the open long enough for protean escape patterns to

17 contribute to their survival. These are the animals for which RTH applies.

\section{Justification of RTH assumptions}

21 We will discuss each of the assumptions of RTH in turn and then will present a computer

22 simulation that illustrates these principles and, at the same time, makes a certain number

23 of predictions. 
The first assumption concerning the ubiquity of predator-prey relations, of course,

2 needs no justification.

3 Concerning the second assumption, we begin with the observation that a great many

4 animal behaviors are hard-wired. These include stereotyped courting and mating

5 behaviors (e.g., Boyce, 1990; Diamond, 1991), aspects of web-building in spiders,

6 cocoon-building in butterflies, nest-building in birds, and even long, complex sequences

7 of seemingly intelligent actions, such as those of the Sphex wasp when it places

8 paralyzed prey in its burrow to provide food for its grubs (Wooldridge, 1965, pp. 82-84).

9 There is, therefore, no a priori reason to assume that escape behaviors would not also

10 depend, at least in part, on reflexive, innate neural control.

11 Bolles (1970) has argued convincingly in favor of species-specific defense reactions

12 (SSDRs). He writes: "Neither the mouse nor the gazelle can afford to learn to avoid;

13 survival is too urgent, the opportunity to learn is too limited and the parameters of the

14 situation make the necessary learning impossible. The animal which survives is one

15 which comes into its environment with defensive reactions already a prominent part of its

16 repertoire." Bolle's point about escape-behavior learning is a consequence, in part, of an

17 obvious, but often overlooked, asymmetry - namely, that the failure on the part of a

18 predator to capture its prey means that it goes hungry, presumably having learned how to

19 be a slightly better predator for its next attack; failure on the part of the prey to escape its

20 pursuer has far more dire consequences. This asymmetry, dubbed the "life-dinner

21 principle" by Dawkins and Krebs (1979), plays a prominent part in the predator-prey

22 simulation described in this paper.

23 A number of examples illustrate Bolles' point. For example, four closely related 
1 species of (asexually reproducing) whiptail lizards in the American Southwest can be

2 distinguished by an experienced observer solely on the basis of their highly stereotyped

3 escape behavior (Schall and Pianka, 1980). In an aquatic environment, Burdick, Harline

4 and Lenz (2007) have shown a similar result for calanoid copepods. Four co-occurring

5 species of these zooplankton can be distinguished by their escape patterns to simulated

6 predators.

$7 \quad$ Arnott, Neil, and Ansell (1999) have shown that for certain predator-approach

8 angles, the brown shrimp (Cragnon cragnon) has a highly predictable escape region. In

9 addition, they showed that there were certain escape regions that these shrimp never use,

10 even though they are physically capable of doing so. Pongráz and Altbäcker (2000) have

11 recently shown that the predatory escape-patterns of European rabbits (Oryctolagus

12 cuniculus) do not require any previous experience with predators, further supporting the

13 idea of innate (i.e., genetically engendered) escape patterns. Zheng et al. (2005) have

14 developed a model based on empirical data of the collective evasion behavior of

15 schooling fish that emerges from innate escape behavior patterns of the individual fish.

16 Domenici and Blake (1993) suggest that angelfish (Pterophyllum eimekei) have innate

17 preferential escape trajectories modulated by sensory feedback. Jablonski (1999) has

18 shown that certain predators take advantage of their prey's stereotyped escape behavior.

19 For example, the painted redstart (Myioborus pictus) uses various displays specifically

20 designed to evoke a hard-wired escape response in its prey, thereby facilitating capture of

21 the prey. Krasne and Wine (1984) discuss the neural correlates of the innate tailflip

22 escape response in crayfish. Eaton (1984) documents the neural mechanisms underlying

23 the startle responses that initiate escape-behaviors in animals ranging from annelids to 
1 mammals. Recently, Domenici, Booth, Blagburn \& Bacon (2008) have shown that inbred

2 (i.e., genetically highly similar) individuals in a population of cockroaches (Periplaneta

3 americana) have a small number of preferred escape trajectories. And finally,

4 anecdotally, the archeologist Louis Leaky (1969) claimed that, as an adolescent, he was

5 able to catch fleeing hares by being able to predict when and in what direction they would

6 jink! We will return to this point in the discussion following the presentation of the

7 simulation results when we discuss the behavioral polymorphisms engendered by the

8 genotype of an individual.

9 Is there an inherent contradiction in our being able to have a measure of predictive

10 accuracy for the escape patterns of a particular species and the claim that individuals

11 within a population have highly variable escape patterns? No, because, while a degree of

12 predictability by us humans, does, indeed, imply reduced variability in escape patterns

13 compared to truly random escape patterns, this still leaves a lot of room for population-

14 level escape-trajectory variability. The degree of variability among individuals must only

15 be enough to outwit predators, which is all that counts. (Outwitting field evolutionists

16 studying animal escape patterns isn't necessary.) In other words, the fact that we humans

17 can detect reduced escape-pattern variability in a particular species of animals does not

18 mean that there is no longer enough variability in these patterns to escape from common

19 predators. A simple example might make this point clear. The escape pattern of rabbits

20 fleeing their burrow involves their making a large circle that returns them to their burrow.

21 So, if all rabbit predators had the cognitive resources of human hunters, they would

22 presumably flush out the rabbit from its hole and simply wait, as hunters do, for its return

23 and then pounce. This is clearly a far more efficient strategy than embarking on a tiring, 
1 energy-intensive chase that might well end up not catching the rabbit. But, presumably,

2 animals other than humans don't have the cognitive facilities to make escape-trajectory

3 predictions that far in the future. As a result, the reduced variability of rabbits' escape

4 trajectories, which allows us humans to make predictions about their escape behaviors, is,

5 nonetheless, usually sufficient to allow them to avoid their common predators. This

6 principle, applied to prey animals in general, is all that is needed for the hypotheses of

7 this paper.

8 Our third assumption is that predators have sufficiently developed neural hardware

9 to allow them to learn from their hunting experiences, both their successes and their

10 failures. Like the first assumption, this one, too, needs little justification. All animals with

11 neural circuitry are capable of learning from their various interactions with the world,

12 including predatory interactions. The capability of predators to predict escape trajectories

13 of prey has often been invoked as the driving force behind the emergence of protean

14 escape behaviors in animals. As Miller (1997) says, “....while the Poker Face [hiding

15 intentions] and KGB [deceit] Strategies remain vulnerable to the coevolution of smarter

16 intention-sensing and deception-foiling capacities, there is no real defense against

17 genuine unpredictability." In short, if prey escape patterns did not vary, predators could,

18 in many cases, learn them, or at least parts of them, thereby gaining a significant adaptive

19 advantage over their prey.

20 It would, therefore, be advantageous for prey to be able to have "unpredictable

21 elements" in their escape response to prevent predators from gaining this advantage. This

22 behavioral unpredictability has been called "protean behavior" (Chance \& Russell, 1959;

23 Driver \& Humphries, 1988; Miller, 1997). Arnott, Neil, \& Ansell (1998), in discussing 
1 escape patterns of the brown shrimp C. crangon, comment that, "Unpredictable elements

2 of the escape response have been shown to be a prominent feature of $C$. crangon escape

3 swimming and are probably important in preventing predators from learning a fixed

4 pattern of response." A detailed chronicle of the near ubiquity in nature of protean escape

5 behavior has been given by Driver and Humphries (1988).

6 The final assumption underlying RTH is that there is an evolutionary cost associated

7 with equipping individuals with the ability to produce protean escape behavior. There are

8 several related issues here. First, the requirement of making choices during escape would

9 be cognitively more costly, and therefore slower, however slightly, than purely reflexive

10 movements. Second, protean behavior in an individual does not come for free. Some

11 neural mechanisms must exist to produce it and those mechanisms would have come at

12 an evolutionary cost. As Driver and Humphries (1988, p. 177) have pointed out,

13 "Organized - through overtly erratic - variations of direction and speed in prey animals,

14 i.e., protean flight, necessitate the evolutionary development of special control

15 mechanisms." The problem is that it is not clear how these mechanisms might work.

16 Driver and Humphries invoke the possibility of mechanisms involving lowered inhibition

17 of motor control neurons and suggest that individual-level protean escape patterns might

18 be generated in a manner similar to epileptic-like convulsions. What is needed, then, is a

19 neural mechanism that would provide feedback-controlled, escape-movement specific,

20 convulsions and it is far from clear how this might work. Further, while epileptic-like

21 convulsions may appear to be random, they may well not be. As Miller (1997) has

22 pointed out, the question of whether animals can actually produce truly random behavior

23 at all is still open to debate. 


\section{Escape behaviors: hard-wired and protean}

2 This brings us to our explanation of how can animals can have escape behaviors that

3 are both hard-wired and protean.

4 Since a given predator rarely, if ever, attacks the same animal twice, nature would

5 not have had to go to the trouble of equipping individuals with any specific,

6 evolutionarily costly, mechanisms for protean escape behavior, as long as there was

7 protean escape behavior at the population level. In short, an individual prey animal is best

8 served by having a largely fixed, reflexive - and therefore, maximally rapid - escape-

9 sequence (or small set of these sequences to be used in different escape contexts), whose

10 unlearnability is ensured because other similar animals have randomly different escape-

11 sequences.

12 This is, of course, not to deny the existence of neurally encoded protean escape

13 behavior in individuals. Rather, RTH suggests that, just as there are polymorphisms for

14 characteristics like cryptic coloration, there are also behavioral (in this case, escape

15 pattern) polymorphisms among individuals. An important challenge for empirical

16 research, then, is to distinguish the extent to which protean escape behavior within a

17 given species is at the individual versus population level.

18 It has been suggested (J. Endler, personal communication) that even if the neural

19 circuitry designed to produce escape patterns was essentially identical for all individuals

20 in a population, minute changes in environmental conditions, hormonal levels, etc. could

21 produce different firing patterns of this circuit that could, in turn, produce radically

22 different, even chaotic, escape behavior, thereby achieving, at essentially no additional

23 cost, the desired protean escape behavior at the individual level. This is certainly 
1 possible, but small neural changes leading to chaotic, or quasi-chaotic, behavior is -

2 fortunately - not a general property of most neural circuitry. Small changes in the

3 environment do not, for example, cause us to speak in a chaotic manner or to act in

4 radically unpredictable ways. Thus, if all individuals did have identical neural escape

5 circuitry, one would have to ask what mechanisms would allow these circuits -- and only

6 these circuits -- to be hyper-sensitive to minute environmental changes, when most neural

7 circuitry is not. Secondly, escape patterns only have to show certain general regularities

8 over time and in certain contexts to allow the predator to learn, however imperfectly,

9 something about its prey escape patterns, thereby giving the predator a significant

10 adaptive advantage over its prey.

12 Prey escape-patterns and their relation to sexual versus asexual reproduction

14 The conundrum of mutation-driven evolution, as Williams (1975) realized, is that

15 evolution, which relies on mutation-driven variability, has evolved myriad mechanisms to

16 keep mutation rates as low as possible. He writes (Williams, 1975, p. 148), “... all

17 observed mutations rates should all be regarded as approximations of zero." This would

18 imply that the innate (i.e., genetically engendered) escape patterns of prey animals would

19 evolve only very slowly if mutations occurring during asexual reproduction were the sole

20 means of modifying them. This would increase the likelihood that predators could learn

21 them. By contrast, sexual reproduction would produce a population of different - and

22 therefore unlearnable by predators - escape patterns at no extra evolutionary cost, while

23 allowing mutation rates to remain as low as possible. 
The simulations presented in the next section compare survival rates of prey under

2 sexual reproduction versus mutation. They will show the clear advantage of sexual

3 reproduction over mutation alone, especially where predation pressure is high. These

4 simulations also show that high mutation rates can, by themselves, generate escape-

5 pattern randomness at the population level. But high mutation rates have other, almost

6 invariably deleterious effects on individuals in the population, which is why nature keeps

7 them as low as possible. In humans, for example, genome mutation rates are on the order

8 of $10^{-8}$ per base pair per generation (Nachman and Crowell, 2000).

\section{Red Tooth Hypothesis Simulations}

12 In what follows, we will present a set of simple predator-prey simulations in which

13 predators are modeled by neural networks designed to learn sequences. Over time these

14 predator networks learn short segments of the prey escape-sequences by successive

15 encounters with the prey. Each instance of pursuit constitutes a learning trial for the

16 predator. In contrast, a prey's failure to escape a predator is (obviously) not a learning

17 experience for the prey. Escape patterns of prey therefore evolve, not during the lifetime

18 of the animal, but over evolutionary time. Each individual in the prey population is

19 modeled as a sequence of "elementary escape-movement (EEM) genes" that determine

20 how it will escape from a predator.

21 We then compare evolution in the prey population achieved through mutation alone

22 with evolution produced by a combination of mutation and crossover. 


\section{Details of the simulation}

2 In all simulations reported here, we begin with an initial population of 100 prey

3 individuals and a single predator. During each generation, we assume that the predator

4 attacks each member of the prey population once, thereby allowing the fitness of each

5 prey individual's escape behavior to be calculated. Reproduction occurs at the end of

6 each generation based on a standard genetic algorithm.

7 We used a standard genetic algorithm (Holland, 1975; Mitchell, 1996) to model the

8 evolution of escape patterns through sexual reproduction. There is a clear description of

9 the algorithm used in Mitchell (1996, pp. 10-12). A population of 100 individuals was

10 maintained at each generation. Single-point crossover was used and both of the newly

11 created offspring were included in the new population. None of the "fittest animals"

12 from the prior generation were explicitly retained in the succeeding generation.

13 We manipulated mutation rates and predator speeds. Each simulation ends either

14 after 30 generations or earlier if the prey population has become extinct. In order to study

15 RTH without, in addition, having to model the dynamics of two interacting populations,

16 we chose to have a single predator which encounters all of the prey each generation.

17 For each simulation the initial population contained 100 prey animals, each with one

18 of two randomly chosen escape-sequence genotypes. A single predator pursued all of the

19 prey individuals during the course of each generation. The fitness of each prey individual

20 was determined by how far it was from the predator after 20 jinks in its escape trajectory.

21 If an animal was caught, it was eliminated from the population. Reproduction was based

22 on fitness with fitter individuals producing more offspring (see Holland, 1975). A new

23 population of 100 individuals was produced at each generation in order to ensure that the 
1 predator had an equal number of learning cycles per generation, thereby allowing the

2 same amount of predator learning across generations. The simulation was stopped after

330 generations or earlier, if all of the prey in the population were caught by the predator.

4

5 Prey

6 Each individual in the prey population is modeled as a string of 80 bits, corresponding to

7 a sequence of twenty 4-bit "EEM genes," each of which codes for a jink angle with

8 respect to the current line of flight. (The length of the escape sequence was arbitrarily

9 chosen and does not affect the conclusions of the RTH simulation.) Each of the 16

10 possible EEM genes corresponds to what Zheng et al. (2005) refer to as fixed (innate)

11 "behavior patterns". The fitness of an escape-sequence, and its corresponding gene

12 sequence, is measured by how far apart the prey with that sequence is from an attacking

13 predator after 20 jinks. Escape patterns for prey that are caught by the predator are

14 assigned a fitness of zero. It seemed reasonable to suppose that the farther an animal was

15 from its pursuer after a given amount of time, the "fitter" its particular escape behavior.

16 For this reason we adopted a continuous measure of fitness, rather than a coarser, all-or-

17 nothing measure of fitness where "fit" corresponded to escape and "unfit" to capture. We

18 did, however, test the all-or-nothing fitness measure and the pattern of results is globally

19 similar to that which is obtained using the continuous measure, the only difference being

20 that the rise in entropy of the population (see Figure 7 and the discussion of entropy)

21 occurs more slowly.

22 In a real environment, of course, escape trajectories are produced by an interaction

23 between underlying behavior patterns and environmental influences on those patterns. 
1 The terrain over which an escape occurs is invariably uneven and strewn with obstacles,

2 such as rocks, trees, and bushes, and no escape pattern would be executed independently

3 of this environment. Animals, even if their escape patterns were completely innate, do not

4 run into trees while escaping. In the interest of simplicity, however, we did not attempt to

5 model these environmental factors. We made the assumption that a given innately-driven

6 escape pattern would give rise to responses in particular environmental contexts that were

7 still learnable. All that is required for predators to improve their prey capture rate is to

8 learn, in general, what its prey is likely to do in a particular context. So, for example, if

9 its prey is approaching a dense thicket, knowing that it will, on average, veer to the right

10 significantly more often than to the left is very valuable -- and learnable -- information

11 for the predator, even if it does not allow for perfect prediction. The response, "veer right

12 when approaching a thicket," we assume to be a product of the underlying escape

13 encoding.

14

15 Predators

16

17 Predators are modeled by a simple three-layer backpropagation neural network whose

18 inputs correspond to the current jink angle of the prey, as well as the previous two jink

19 angles, which provide context information. The network attempts to predict the next jink

20 angle of the prey. If it is able to accurately predict the upcoming jink angle, it

21 stochastically begins to make attempts to learn a two-jink prediction. If it is able to learn

22 this, as well, it attempts a three-jink prediction.

23 The network, therefore, has 12 input nodes (plus bias nodes on the input and hidden 
1 layers), corresponding to the present jink angle and the previous two jink angles. There

2 are 12 hidden nodes. There are also 12 output nodes, the first four of which correspond to

3 the prediction of the next jink, the second set of four corresponds to a two-jink prediction,

4 and the final group of four corresponds to a three-jink prediction. The hidden layer is

5 fully connected to the first set of four output nodes (representing the immediately

6 following jink angle), fully connected to the second set of output nodes (representing a

7 two-jink prediction), and fully connected to the third set of output nodes (representing a

8 three-jink prediction). The learning rate was 0.01 and momentum 0.9. Both the hidden

9 and output layers were fed activation from a bias node in the preceding layer. The hidden

10 and output layers used standard sigmoid squashing functions. A standard sum of squares

11 error function on output was used to drive learning. A generalized delta learning rule was

12 used for all weight changes. (See Rumelhart \& McClelland, 1986, vol. 1, pp. $45-54$ for

13 implementation details for a standard backpropagation neural network.)

14 Initially, the network attempts to predict the next jink of the prey. As it gets better

15 and better at doing this (i.e., when the network error is low for the next jink in the

16 sequence), it will (stochastically) start to make two-jink predictions. If it gets good at

17 two-jink predictions, it will try to make three-jink predictions. The better the predator's

18 predictive look-ahead is, the more likely it will be to catch its prey. The less of any given

19 individual prey's escape-sequence it can predict, the harder it will be to catch that

20 individual.

21 The predator pursues all 100 individuals in each generation of prey. The learning

22 acquired during previous generations remains with the predator as it pursues prey in

23 future generations. In other words, the predator will have a total of 3000 learning 
1 experiences during the course of the simulation. (This corresponds to 3000 learning

2 "epochs" of the neural network that constitutes the predator.)

3 Figure 1 shows a typical predator-prey chase. After 20 jinks, the distance remaining

4 between the predator and its prey is used as a measure of the fitness of the prey's escape-

5 sequence. If the predator catches the prey before the end of the escape sequence, the

6 fitness of the sequence is set to 0 .

$<$ Figure 1 here $>$

9 Figure 1. A typical predator-prey chase. The fitness of the escape-trajectory is

10 determined by the distance between predator and prey after 20 jinks.

12 In a real predator-prey interaction, a number of factors are crucial in determining the

13 probability that the prey will escape, most importantly, the animals' maximum speed,

14 acceleration, maneuverability, and endurance (Alexander, 2003). In the present

15 simulation we chose to model only one of these parameters, maximum speed. We felt that

16 the inclusion of the other variables was unnecessary to make the main point of the paper

17 - namely, the advantages of sexual reproduction in producing the emergence of protean

18 escape patterns at the population level which hinder the predator from catching its prey.

19 In the constant evolutionary arm-races between species of prey and their predators,

20 the maximum speed of a given predator is generally not too far above that of its prey

21 (Alexander, 2003). For this reason, the ratio of prey-to-predator speeds in our simulations

22 varies from 1:1.1 to $1: 1.5$.

23 In our simulations, we did not explicitly model the differences in the centripetal 
1 forces involved when a larger predator turns compared to its smaller prey. This

2 difference in forces means that, at close quarters, it is inherently advantageous for prey to

3 turn tightly (Alexander, 2003) to avoid a heavier predator. The main points of our model

4 are about prediction and learning on the part of the predator and evolution on the part of

5 the prey and, for this reason, we made no assumptions about the relative size of the

6 predators and prey. As a result, the forces involved in turning were not modeled

7 explicitly. However, there is no a priori reason that this could not have been done. Had

8 we done so, it is likely that we would have seen the systematic emergence of tight turning

9 behavior when the predator is close to the prey.

\section{Predator and prey position calculations}

13 Predators begin their pursuit at the origin of a grid. Each prey individual starts at $(0,2)$ on

14 that grid. Prey are assumed to have a speed of 1 at all times. Predators have a fixed

15 speed that is some factor of the prey's speed (e.g., 1.1, 1.2, 1.5). A fixed "reaction time"

16 for the predator is associated with each jink of the prey. If the predator correctly predicts

17 the next jink direction, this reaction time is decreased by a fixed amount. The predator

18 predicts where its prey will go on its next jink (or jinks) and heads in the direction of its

19 prediction. Given that we know the current position of the predator, its direction, its

20 speed, and its time to react to the prey's jink, we can calculate its next position. As the

21 predator learns and gets better at one-jink predictions, it will (stochastically) occasionally

22 make two-jink predictions and, ultimately, sometimes, three-jink predictions (directly

23 heading towards the spot the prey will be after two or three jinks). From its starting 
1 point, the prey simply executes the fixed escape trajectory defined by its sequence of

2 twenty EEM genes.

4 Evolution of survival rates for sexually versus asexually reproducing populations 5

6 In what follows we compare survival rates in sexual versus asexual populations. We will

7 begin by showing (Figure 2) survival-rate curves over 30 generations for a given predator

8 pursuit speed (in this case, 1.4, compared to the prey's speed of 1) and a given mutation

9 rate $(\mu=0.0001)$.

10 Figure 2 shows median and average survival rates for sexually and asexually

11 reproducing populations when the prey-predator speed ratio is $1: 1.4$. The data were

12 averaged over 20 runs. The average data was calculated only for non-extinct populations

13 at each generation (i.e., if, say, at generation 23 there were only five runs in which the

14 prey population had survived, the average survival rate for that generation was calculated

15 based on those five populations).

18 Figure 2. Evolution of survival rates of sexual and asexual prey populations over 30

19 generations (average and median data)

21 A key result of our simulations is illustrated in Figure 2. We have a low mutation rate of

$22 \mu=0.0001$ and a prey-predator speed ratio of 1:1.4. Under these conditions, the median

23 survival rate for the sexually reproducing population climbs rapidly to above $80 \%$ and 
1 levels out around 90\%. In contrast the median survival rates for asexual populations drop

2 to 0 by 20 generations. In other words, most of the time they will become extinct before

330 generations.

4

5 Survival rates, mutation rates, and predation pressure

6

7 What happens if we vary mutation rates and predation pressure? The graphs in Figures

$83 \mathrm{a}, 3 \mathrm{~b}$, and $3 \mathrm{c}$ show that if predation pressure is low (i.e, predators run only $10 \%$ faster

9 than their prey, Figure 3a), low mutation rates can be maintained in both sexual and

10 asexual populations and prey survival rates will remain high. However, as predation

11 pressure increases (up to a prey-predator speed ratio of 1:1.5 in Figure 3c), sexual

12 reproduction becomes essential to simultaneously maintain both low mutation rates and

13 high prey survival rates. Of course, high mutation rates in asexual populations also

14 produce the variety of escape patterns that sexual reproduction produces at low mutation

15 rates. Even with high predation pressure (prey-predator speed ratio $=1: 1.5$ ), if the base

16 mutation rate of 0.0001 is increased by a factor of 50 to 0.005 , there is no difference in

17 survival rates of asexual and sexual populations. But of course, high mutation rates

18 almost invariably have other deleterious effects on the organisms, as we have discussed

19 above.

20 In the original population, if the two original escape-sequence genotypes differ only

21 slightly (e.g., by no more than $20 \%$, rather than both being random), we obtain essentially

22 the same results as in Figures 3a, 3b, and 3c. 
10 In short, for environments of low predation pressure, asexual reproduction is as

11 effective as sexual reproduction in producing effective polymorphic escape-behavior.

12 However, under heavy predation pressure, sexual reproduction becomes much more

13 important. This corresponds to what is commonly observed in certain heterogamous

14 animals. For example, the freshwater crustacean Daphnia generally reproduces

15 parthenogenically, then switches to sexual reproduction as competition and predation

16 increase. Williams (1975) and Bell (1982) document numerous cases of this shift to

17 sexual reproduction when the environmental conditions, such as climate, increased

18 predation, etc., become harsher.

19

\section{$20 \quad$ Prey-predator first encounters}

22 Our simulations (Figure 4) also show that when a founder population of prey animals first

23 encounters a new predator, initially, the predators will kill a significant number of them,

24 frequently leading to the extinction of the prey. However, if extinction does not occur in 
1 the first few generations, the counterbalancing effects of sexual reproduction producing a

2 wide variety of prey escape trajectories will rapidly become evident. Two things happen:

3 i) prey with better escape-sequences survive the initial onslaught of the predator and

4 transmit to future generations their more effective escape-sequences and ii) more escape

5 sequences are generated, thus making it impossible for predators to learn prey escape

6 movements.

7 In simulations with asexually reproducing populations with a low mutation rate and

8 low predation pressure, if the population does not go extinct early on, we see the same

9 general evolution of survival rates, only with a much more gradual slope. In other words,

10 asexual populations are more vulnerable than sexual populations to initial predatory

11 attacks, but if the population manages to survive long enough, the effects of mutation

12 alone will ultimately be the same as those of recombination - namely, the population-

13 level escape trajectories will become varied enough to afford individual-level protection

14 from the predator.

$<$ Figure 4 here $>$

17 Figure 4. Prey are initially vulnerable to attack by predators learning their escape-

18 behaviors. Evolutionary forces gradually reduce the effectiveness of these attacks.

20 Sexual recombination of escape-sequences makes the predator's chase harder

22 Figure 5 compares how easy or difficult it is for a predator to follow its prey. We assume

23 that the prey will jink 20 times in its escape-sequence. The predator pursues all 100 of 
1 prey in each generation once, which gives a total of 3000 encounters with prey. This

2 means that the neural network that models the predator has 3000 learning cycles to

3 predict where the prey will be after each jink. Accurately predicting where the prey will

4 be means that the predator can take shortcuts during pursuit of the prey. The graph

5 compares the evolution of the number of direction changes that the predator needs to

6 make when pursuing asexually reproducing prey compared to sexually reproducing prey.

7 After 3000 learning cycles, the predator requires only 10 changes of direction to

8 successfully track its prey, whereas for a sexually evolving prey population, where

9 population-level protean escape-behavior is established, very little anticipatory prediction

10 (i.e., learning of escape patterns) is possible and the predator requires an average of 17 to

1118 changes of direction to pursue its prey.

\section{$<$ Figure 5 here $>$}

14 Figure 5. The evolution of the number of changes of direction during pursuit of its prey in

15 populations of asexually and sexually reproducing prey. For 20 jinks of the prey in an

16 asexual population the predator needs only 10 changes of direction, whereas 17 to 18 are

17 required for when pursuing sexually producing prey.

\section{Protean escape-patterns versus good escape patterns}

21 An escaping animal can be protected in at least two different ways when fleeing an

22 attacker. It can either have an excellent escape-sequence that is intrinsically hard for the

23 predator to follow, even taking into consideration the predator's ability to learn segments of 
1 it. Or, even if its escape-sequence is only moderately effective by itself, it can be protected

2 by the fact that the predator cannot predict its escape-patterns because of the random nature

3 of escape patterns at the population level. This would imply that in sexually reproducing

4 species individual protection is likely to be provided by a great many escape patterns of

5 varying intrinsic quality. By contrast, in asexual populations there will be less escape-

6 pattern entropy at the population level, which will mean that, in general, (parts of) these

7 escape sequences will be easier to learn by the predator. Consequently, those asexual

8 populations that do survive will have escape sequences that, even though parts of them can

9 be learned by the predator, are, nonetheless, still good enough to escape the predator. This

10 implies that escape trajectories of asexual populations will have to be, on average,

11 intrinsically better than those of sexual populations under equivalent predation pressure.

\section{Variability of escape patterns in sexual and asexual populations}

14 In Figures $6 \mathrm{a}$ and $6 \mathrm{~b}$ we can see the results of a simulation where the mutation rate

15 was 0.0001 and the predator-to-prey maximum speed ratio was 1:1.4. It is clear that in the

16 case of the sexually reproducing population (Figure 6b), the range of escape-patterns is

17 considerably more varied than in the asexually reproducing population (Figure 6a).

$<$ Figure 6a here $>$

20 Figure 6a. A random selection of 36 escape-patterns in an asexually reproducing population

21 with a mutation rate of 0.0001 after 30 generations. 
1 Figure 6 b. A random selection of 36 escape-patterns in a sexually reproducing population

2 with a mutation rate of 0.0001 after 30 generations.

3

4 A quantitative measure of population-level escape-trajectory variability

It seems clear from a visual examination of Figures $6 \mathrm{a}$ and $6 \mathrm{~b}$ that after 30 generations sexually evolving populations have more highly varied escape trajectories than asexually evolving populations. But it would be very useful to have a measure of

9 this variability. In particular, this would allow us to trace the evolution of variability of

10 escape trajectories within a population over time. If RTH is correct, this evolution of

11 variability should correlate closely with the evolution of prey survival rates.

12 To measure population-level escape-trajectory variability, we used a standard

13 conditional entropy measure (MacKay, 2002) taken from information theory.

14 Conditional entropy is calculated as follows:

$$
H(S)=-\sum_{i \in I} p(i) \sum_{j} p(j \mid i) \log _{2} p(j \mid i)
$$

16 In our simulations this measure is interpreted as follows. For a given population S, we

17 consider a set of randomly chosen escape trajectories (in this case, the escape trajectories

18 of 36 individuals in the population). Since there are 16 possible jink angles and the

19 predator uses the most recent jink, along with the two previous jinks before that, to

20 predict the upcoming jink, this means that there $16^{3}=4096$ different "jink contexts".

21 However, in fact, there are only a relatively limited number of different jink contexts

22 encountered by the predator (the fewer that are actually encountered, the lower the

23 variability of the population of escape-trajectories). The probability of each of the jink 
1 context actually encountered (indicated by $i \in I$ in the entropy formula) with respect to

2 all possible jink contexts is indicated in the formula above by $p(i)$. The conditional

3 probability of the upcoming jink angle, $j$, given the context, $i$, is designated by $p(j \mid i)$.

4 We calculate these values over the set of all escape trajectories in our sample population

5 to arrive at an entropy value of $\mathrm{H}_{1}$ for single-jinks, $\mathrm{H}_{2}$ for double-jinks, and $\mathrm{H}_{3}$ for triple-

6 jinks. The final value $\mathrm{H}$ for the population of escape trajectories is the average of these

7 three values.

$<$ Insert Figure 7 here. $>$

11 Figure 7. Evolution of the entropy (i.e., randomness) of escape trajectories of individuals

12 from asexually reproducing and sexually reproducing populations.

14 Figure 7 is a graph of the evolution of $\mathrm{H}$ at each generation. In particular, this graph

15 illustrates the rapidity with which sexual evolution creates variability in the escape

16 trajectories at the population level compared to asexual evolution. In the first 5-7

17 generations, the entropy of the sexual population's escape trajectories (i.e., SEX 0.0001)

18 increases rapidly and by 12 generations it has reached its maximum variability. By

19 contrast, the entropy of the escape trajectories of the asexual population with the same

20 mutation rate (i.e., ASEX 0.0001) increases only very slowly over the course of the 30

21 generations of the simulation. These values are drawn from a population where the

22 predator is only slightly faster than the prey (predator-prey speed ratio: 1.1) with a low

23 rate of mutation $(\mu=0.0001)$. In this way, very few populations went extinct during any 
1 given run of the simulation. The graph also shows that as the mutation rates in asexual

2 populations increase from 0.0001 to 0.005 (i.e., a 50-fold increase), the escape-trajectory

3 entropy curves approach those of a sexually reproducing population with a mutation rate

4 of $\mu=0.0001$. These graphs show how sexual reproduction can ensure high variability

5 at the population level of escape trajectories, all the while keeping the mutation rates very

6 low.

7

8 Discussion

9 The simulations presented in this paper show that, if there are underlying innate behavior

10 patterns comprising escape behavior, then sexual reproduction would be a more effective

11 means than asexual reproduction of producing protean escape behavior at the population

12 level, while allowing faster, reflexive behavior to be maintained at the individual level. In

13 other words, evolution may well have endowed individuals with neural mechanisms to

14 produce random behavior, but this would have been a far more evolutionarily costly

15 process than producing this behavior at the population level. This is not to deny the

16 existence of individual-level protean behavior, of course, but it is hard to see what forces

17 would have caused evolution to develop it in individuals if, in fact, equivalent protection

18 from predators had already been achieved by population protean behavior that emerged

19 from sexual reproduction. It could have been that sexual reproduction was evolution's

20 response to the need for rapid emergence of population-level protean escape behaviors. In

21 any event, once sexual reproduction was in place, generating protean escape-behavior at

22 the population level would have incurred almost no extra cost to prey but would have

23 provided significant adaptive advantages. 
It would be interesting to attempt to tease apart the extent to which observed protean

2 escape-behaviors are individual-level behaviors or population-level polymorphic

3 behaviors. Almost all studies of escape behaviors to date have been done on groups of

4 individuals. It would be interesting to begin with two animals with an identical genetic

5 makeup (i.e., identical twins), raised from conception in an identical environment and

6 then placed in an experimental apparatus similar to that used by Arnott et al. (1999) in

7 order to determine the extent to which their respective escape behaviors differed. This

8 would give us a better idea of the degree to which escape behaviors are innately

9 engendered. Empirical studies of this kind on a small naturally occurring clonal fish

10 species Kryptolebias marmoratus are currently under way in Britain in the Animal

11 Behaviour Group in the Psychology Department of the University of Exeter (D. Croft, 12 personal communication).

13 Crow \& Kimura (1965) and Maynard-Smith (1971) proved that one of the fundamental

14 flaws with theories that suggested that sexual reproduction resulted in increased rates of

15 evolutionary adaptation through gene accumulation was that they only applied to

16 populations whose size approached infinity. For small populations, for example,

17 populations with less than a thousand individuals, they showed there was essentially no

18 advantage to gene accumulation through sexual reproduction.

19 But as the above RTH simulations show, this does not apply to the hypothetical jink

20 genes. This is because mutations of these genes, unlike most genes, do not have deleterious

21 consequences for the individual in which they occur. On the contrary, their expression

22 simply leads to a different escape-trajectory in that individual. This contributes to

23 population-level protean escape behavior which protects not only the individual in which 
1 the mutation occurred, but the other individuals in the population.

2 In other words, if EEM genes that are linked to elementary escape-behavior patterns

3 do, indeed, exist then the mutations of these genes and their subsequent accumulation and

4 recombination through sexual reproduction will be highly advantageous to individual prey,

5 however small the population.

6 Our simulations demonstrate a manner in which learning can drive evolution.

7 Further, RTH gives rise to a number of predictions, such as, the entropy of prey's

8 population-level escape-patterns should be proportional to the intelligence of their

9 predators; the diversity of escape patterns should tend to be larger in sexually

10 reproducing populations, the diversity of escape patterns in asexually producing animals

11 should be smaller, but they should be of better quality; the greater the predation pressure,

12 the more important and prevalent recombination by sexual reproduction becomes; etc.

13 Although we have not modeled this here, it is clear that simulations of this sort

14 could, and should, be extended to include the co-evolution of multiple generations of

15 predators. The quasi-impossibility of learning the escape behavior of its prey because of

16 its population-level protean nature would presumably stymie the effects of Baldwin-like

17 learning (Baldwin, 1896; Morgan, 1896; Hinton \& Nowlan, 1987; French \& Messinger,

18 1994) on the predator genome. In other words, the impossibility of learning during

19 hunting would have meant that hunting would not have driven improved learning

20 capacities in predators over evolutionary time. This might help explain why “...true co-

21 evolution ... seems remarkably rare in predator-prey systems." (Endler, 1991) Once a

22 sufficient degree of randomness in escape behavior was achieved (either at the individual

23 or at the population level), the predator would no longer be able to learn prey escape 
1 patterns from prior encounters with that prey.

2 Empirical tests using heterogamous animals, similar to those designed to explore

3 RQH (e.g., Lively, 1987), could be run for RTH as well. RTH would, for example,

4 predict that heterogamous animals in asexual reproductive mode would tend to switch to

5 sexual reproduction when the predator pressure in their environment increased,

6 something which is known to be the case for the freshwater crustacean Daphnia (Bell,

7 1982). This water flea reproduces by parthenogenesis then switches to sexual

8 reproduction as competition and predation increase.

9 In the simulations presented in this paper there is a one-to-one correspondence

10 between EEM genes and their expression as primitive escape-movements. Sequential

11 combinations of these primitive escape movements are assumed to determine more

12 complex escape patterns. However, it may be that other genes regulate these EEM genes,

13 whose expression would, therefore, not be identical in all animals of the species. This

14 does not, however, affect the central point of the paper - namely, that each animal will

15 have a small number of escape patterns that are fixed (i.e., innate) and that the degree of

16 similarity of these patterns across the population determines how learnable they are by

17 predators. But regardless of how the genotype-phenotype mapping is achieved, RTH

18 suggests that the least costly way for evolution to produce unlearnable individual-level

19 escape patterns is to ensure the randomness of these escape sequences at the population

20 level via sexual recombination.

\section{Conclusion}


1 The goal of this paper has been to suggest that, given the ubiquity of predator-prey

2 relations in nature, sexual reproduction would likely produce polymorphic escape

3 behavior more rapidly and more effectively than asexual reproduction. This leads to the

4 suggestion that protean escape behavior, heretofore taken to be an individual-level

5 phenomenon, is, to a significant extent, actually population-level behavior.

6

8 Acknowledgments

10 This work was supported in part by grants FP6-NEST-029088 and FP6-NEST-516542

11 from the European Commission. Thanks to Gray Clossman, Darren Croft, John Endler,

12 Stephen Lea, Joah Madden, Michael Thomas, and Mark Weaver for their contributions to

13 the ideas and emphasis of this paper. Particular thanks to three anonymous reviewers and

14 the editor for their careful reading of this paper and their insightful comments on how to

15 improve it. The author would also like to thank the Firestone Library at Princeton

16 University for permission to use their collections.

\section{References}

20 Alexander, M. R. (2003). Principles of Animal Locomotion. Princeton, NJ: Princeton

21 University Press.

22 Baldwin, J. M. (1896). A new factor in evolution. American Naturalist, 30:441-451,

23 LEA. $335-340$. 
1 Bell, G. (1982). The Masterpiece of Nature: The evolution and genetics of sexuality.

2 Berkeley, CA: University of California Press.

3 Boyce, M. S. (1990). The Red Queen visits sage grouse leks. American Zoologist, 30, $4 \quad 263-270$.

5 Burt, A. and Bell, G. (1987). Mammalian chiasma frequencies as a test of two theories of 6 recombination. Nature 326, $803-805$

7 Chance, M.R.A. \& Russell, W.M.S. (1959). Protean displays: a form of allaesthetic 8 behaviour. Proceedings of the zoological society of London, 132, 65-70.

9 Crow, J. F., and Kimura, M. (1965). Evolution in sexual and asexual populations. 10 American Naturalist, 99, 439-450.

11 Dawkins, R. and Krebs, J. R. (1979). Arms Races Between and Within Species, 12 Proceedings of the Royal Society of London, Series B, Biological Sciences, $13 \quad 205: 1161,489-511$

14 Diamond, J. M. (1991). Borrowed sexual ornaments. Nature, 349, 105.

15 Domenici, P., Booth, D., Blagburn, J. M., \& Bacon, J. P. (2008). Cockroaches keep

16 predators guessing by using preferred escape trajectories. Current Biology 18, 1792$17 \quad 1796$.

18 Driver, P. M., and Humphries, N. (1988). Protean behavior: The biology of 19 unpredictability. Oxford U. Press.

20 Eaton, R. C. (1984). Neural Mechanisms of Startle Response. NY: Plenum Press.

21 Endler, J. (1991). Interactions between predators and prey. In J.R. Krebs and N. B.

22 Davies (eds.) Behavioural ecology: An evolutionary approach, $3^{\text {rd }}$ edition. Oxford, 23 UK: Blackwell. 
1 Fisher, R. A. (1930). The genetical theory of natural selection. Oxford University Press.

2 French, R. M. and Messinger, A. (1994). Genes, Phenes and the Baldwin Effect:

3 Learning and Evolution in a Simulated Population. In Brooks, R. and Maes, P.

4 (eds.). Artificial Life IV. Cambridge, MA: MIT Press, 277-282.

5 Hamilton, W. D. (1975). Extraordinary sex ratios. Science, 156, 477-488.

6 Hamilton, W. D. (1980). Sex versus non-sex versus parasite. Oikus, 35, 282-290.

7 Hinton, G. E. and Nowlan, S. J. (1987). How learning can guide evolution. Complex

$8 \quad$ Systems, 1:495-502.

9 Holland, J. H. (1975), Adaptation in Natural and Artificial Systems, Ann Arbor, MI:

10 University of Michigan Press.

11 Howard, R. S. \& Lively, C. M. (1994). Parasitism, mutation accumulation and the

12 maintenance of sex. Nature, 367:554-557

13 Howard, R. S. \& Lively, C. M. (2003). The ratchet and the Red Queen: the maintenance

14 of sex in parasites. Journal of Evolutionary Biology, 15, 648-656.

15 Jaenike, J. (1978). A hypothesis to account for the maintenance of sex with populations.

16 Evolutionary Theory, 3, 191-194.

17 Krasne, F. and Wine, J. (1984). The production of crayfish tailflip escape responses. In R.

18 C. Eaton (ed.) Neural Mechanisms of Startle Response, 179-211.

19 Leaky, L. S. B. (1969). Animals of East Africa. National Geographic Society,

20 Washington, D.C.

21 Levin, D. A. (1975). Pest pressure and recombination systems in plants. American

$22 \quad$ Naturalist, 109. 437-451.

23 Lively, C. M. (1987). Evidence from a New Zealand snail for the maintenance of sex by 
parasitism. Nature 328, 519-521.

2 MacKay, D. (2002). Information Theory, Inference and Learning Algorithms. Cambridge

3 University Press.

4 Maynard Smith, J. (1971). What use is sex? Journal of Theoretical Biology, 30, 319-335.

5 Maynard Smith, J. (1976). A comment on the Red Queen. American Naturalist, 110, 325$6 \quad 330$.

7 Maynard Smith, J. (1978). The evolution of sex. Cambridge University Press.

8 Miller, G. F. (1997). Protean primates: The evolution of adaptive unpredictability in

9 competition and courtship. In A. Whiten \& R. W. Byrne (Eds.), Machiavellian

10 Intelligence II: Extensions and evaluations (pp. 312-340). Cambridge University

11 Press.

12 Mitchell, M. (1996), An Introduction to Genetic Algorithms, MIT Press, Cambridge, MA.

13 Morgan, L. On modi variation. variation. Science 4:733-40 (1896).

14 Muller, H. J. (1932). Some genetic aspects of sex. American Naturalist, 66, 118-138.

15 Nachman, M.W., Crowell, S. L., (2000). Estimate of the mutation rate per nucleotide in

16 humans. Genetics, 156, 297-304.

17 Pongráz, P. \& Altbäcker, V. (2000). Ontogeny of the responses of European rabbits

18 (Oryctolagus cuniculus) to aerial and ground predators. Canadian Journal of

19 Zoology, 78(4): 655-665.

20 Rumelhart \& McClelland and the PDP Research Group (1986), Parallel Distributed

21 Processing: Explorations in the Microstructure of Cognition, vol. 1, Cambridge,

22 MA: The MIT Press.

23 Schall, J. J. \& Pianka, E. R. (1980). Evolution of escape behavior diversity. The 
American Naturalist, 115(4), 551-566.

2 Stenseth, N. C. and Maynard Smith, J. (1984). Coevolution in ecosystems: Red Queen 3 evolution or stasis? Evolution, 38(4), 870-880.

4 Van Valen, L. (1973). A new evolutionary law. Evolutionary Theory, 1, 1-30.

5 Williams, G. C. (1975). Sex and evolution. In Monographs in Population Biology.

6 Princeton: Princeton University Press.

7 Wooldridge, D. E. (1963). The Machinery of the Brain. NYC: McGraw-Hill Publishers.

8 Zheng, M., Kashimori, Y., Hoshino, O., Fujita, K., Kambara, T. (2005). Behavior pattern

9 (innate action) of individuals in fish schools generating efficient collective evasion 10 from predation. Journal of Theoretical Biology, 235, 153-167. 


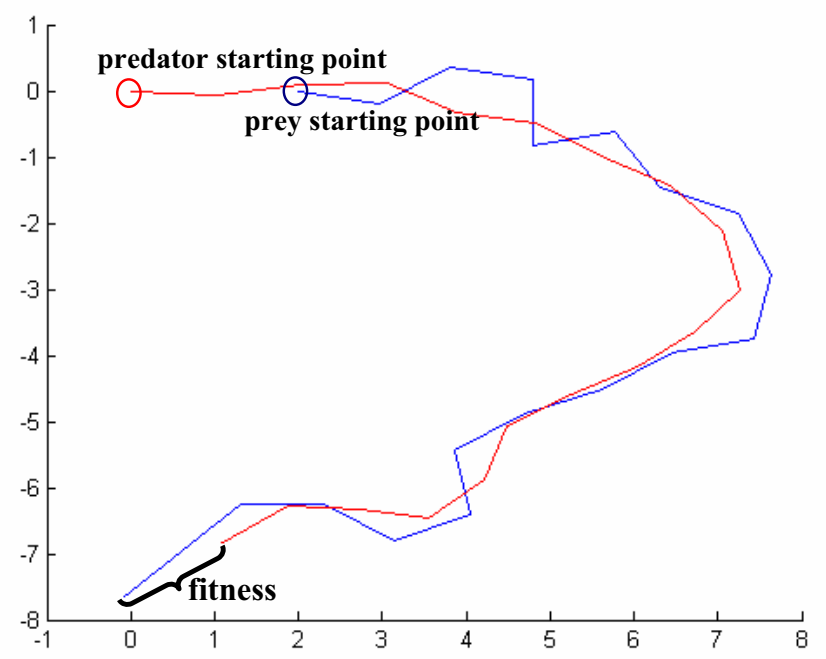

Figure 1 


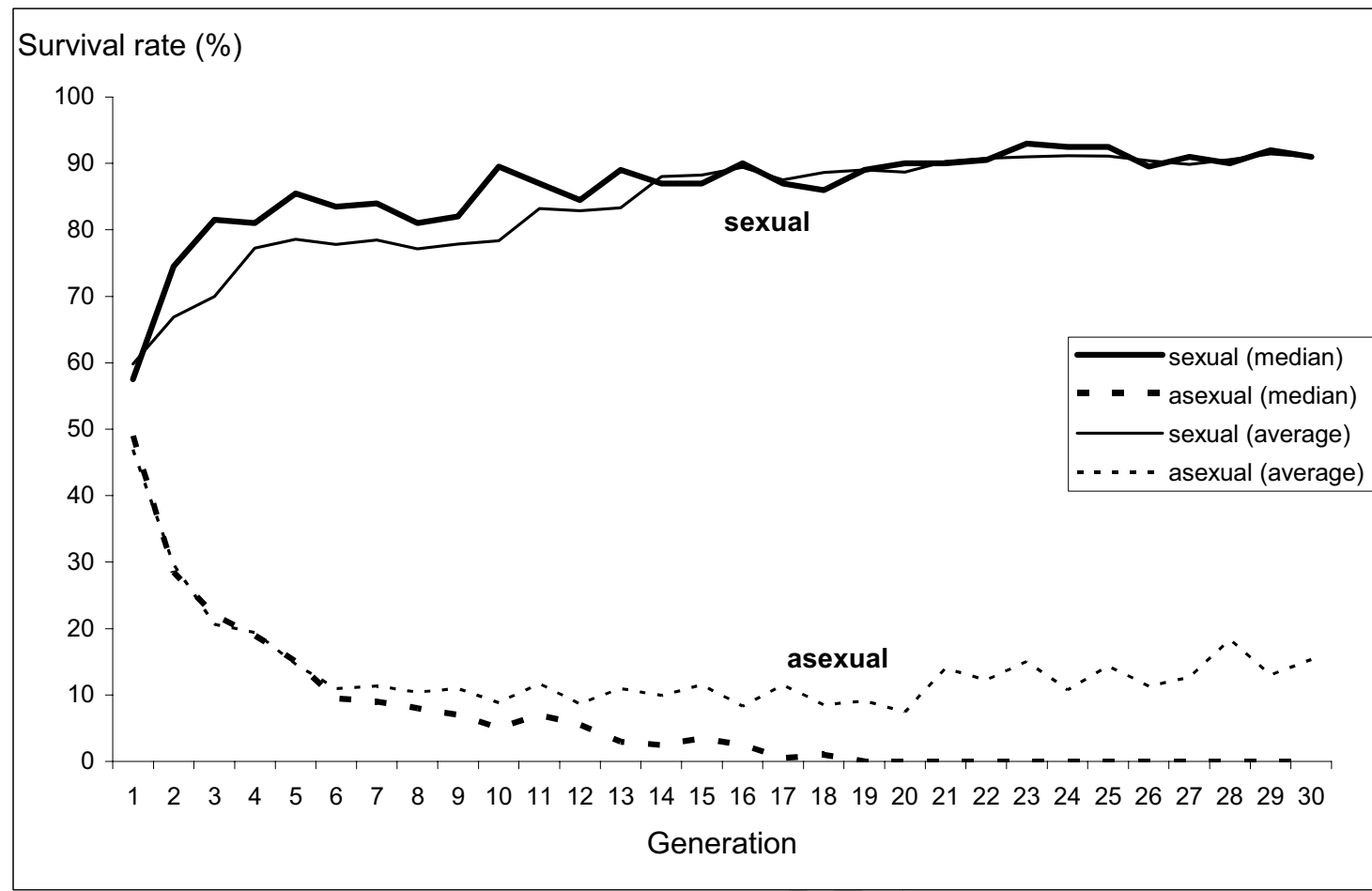

Figure 2. 


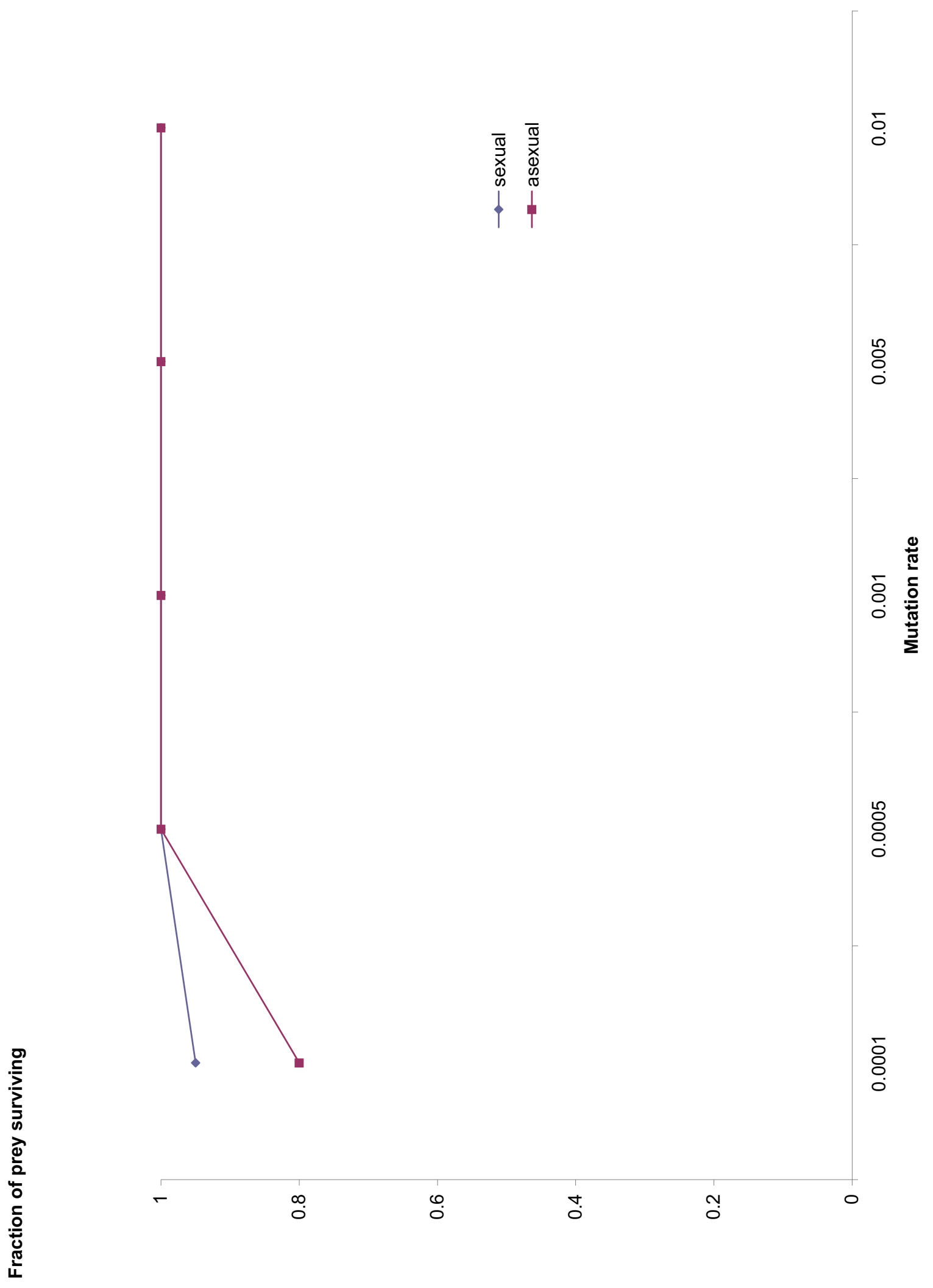




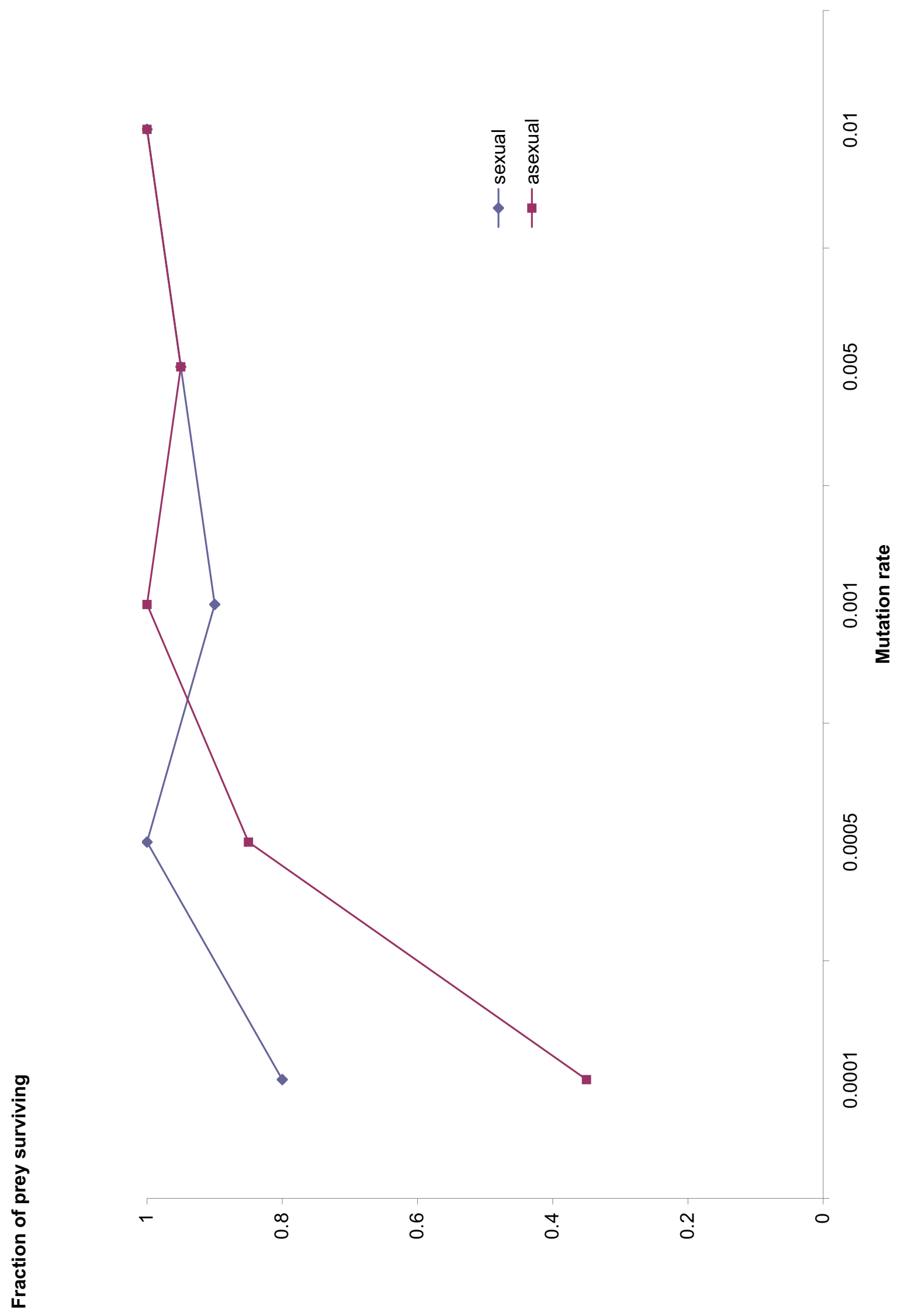




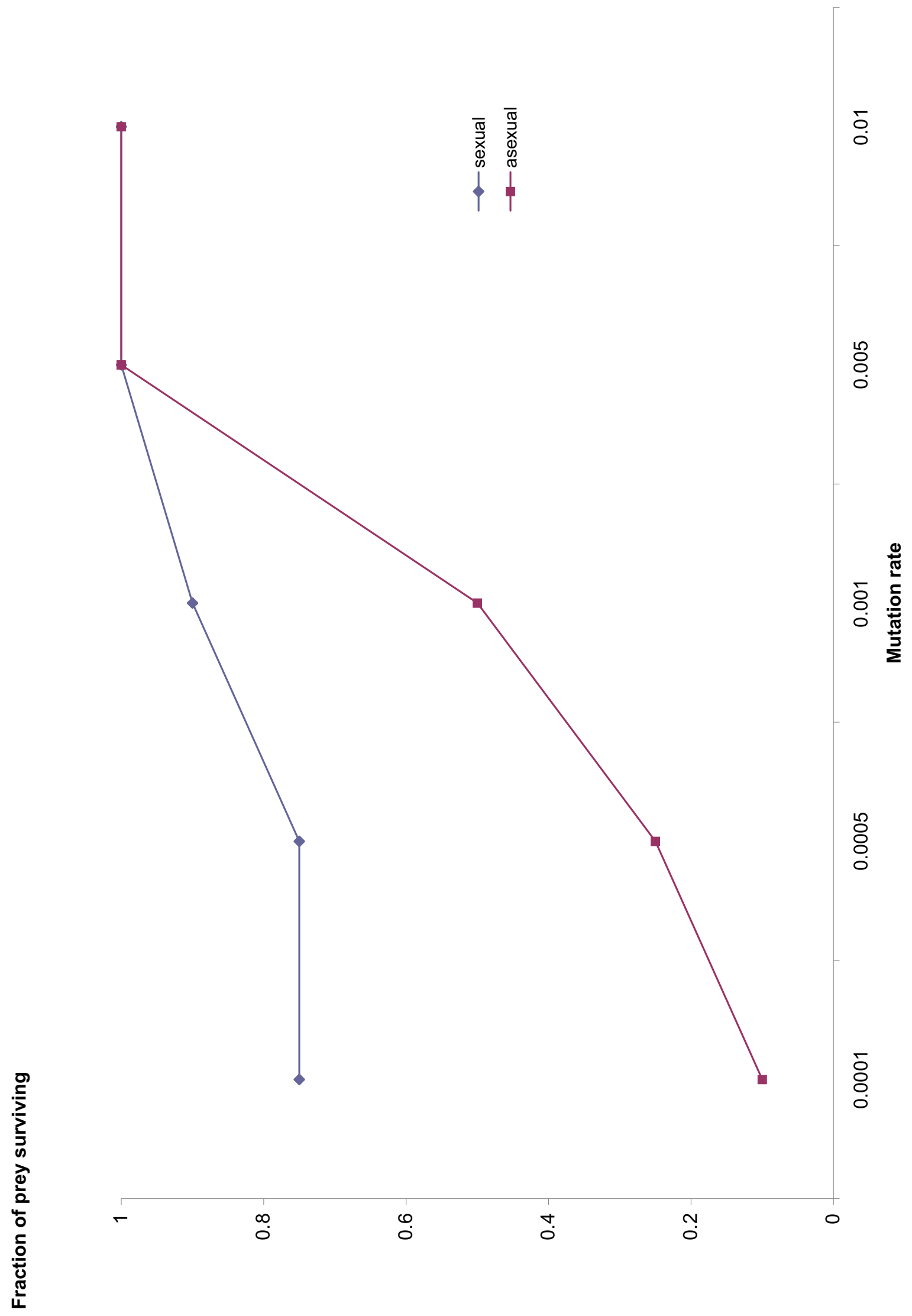




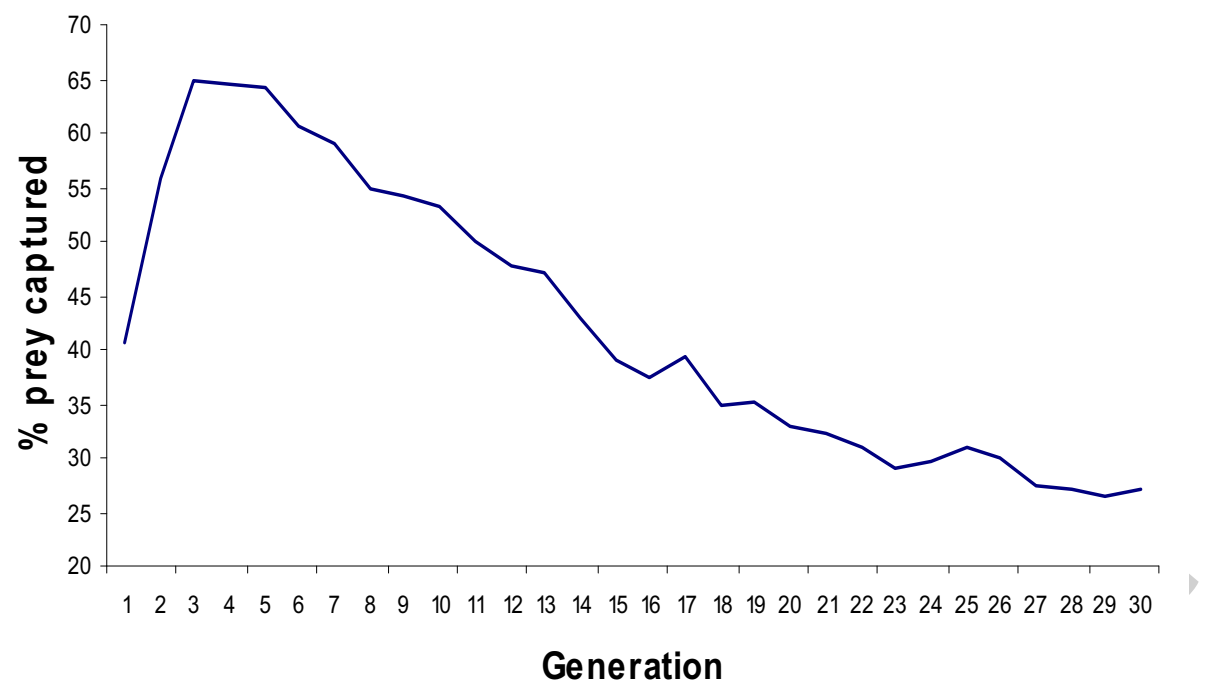

Figure 4 
No. of predator

direction changes

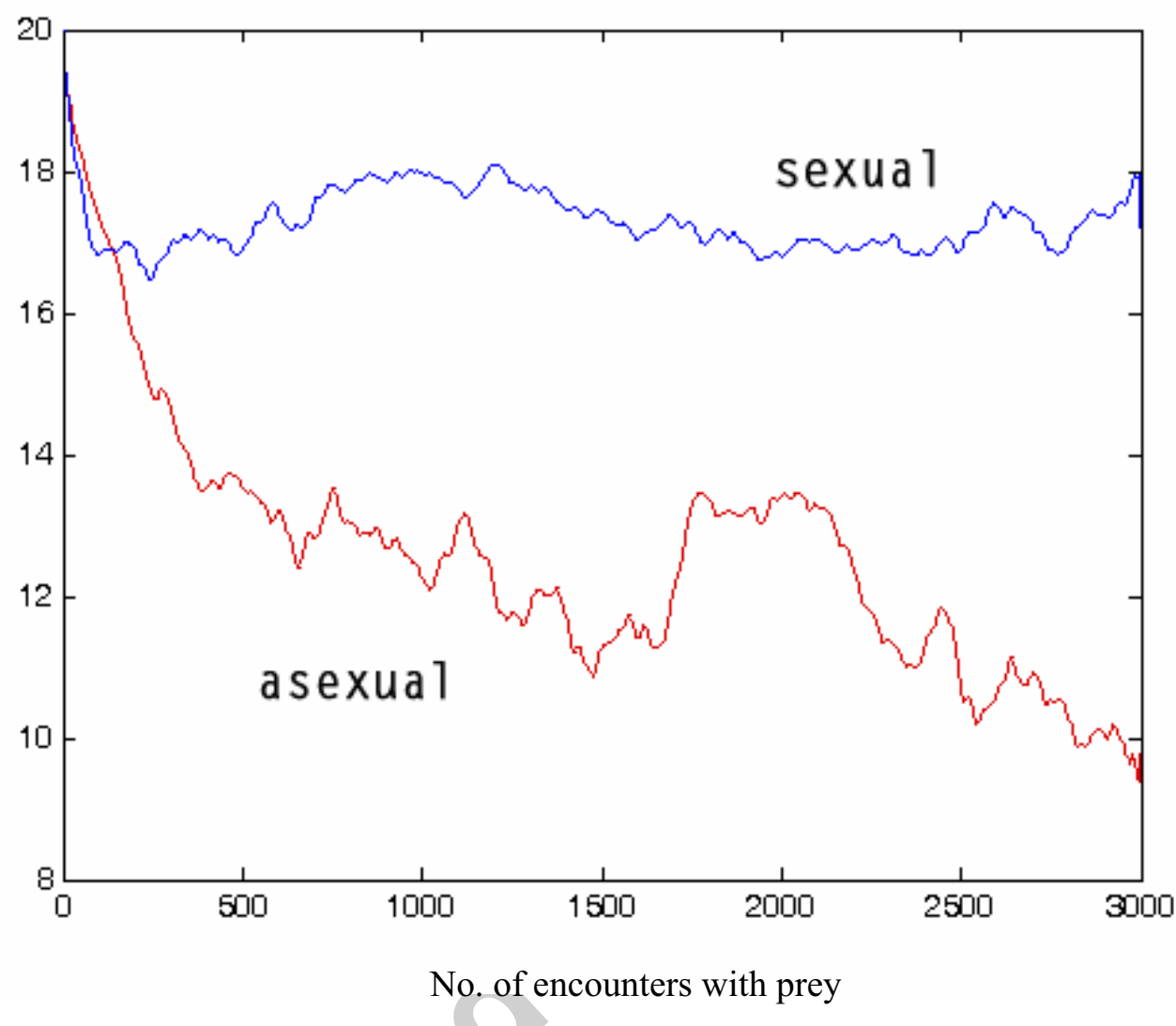

Figure 5 

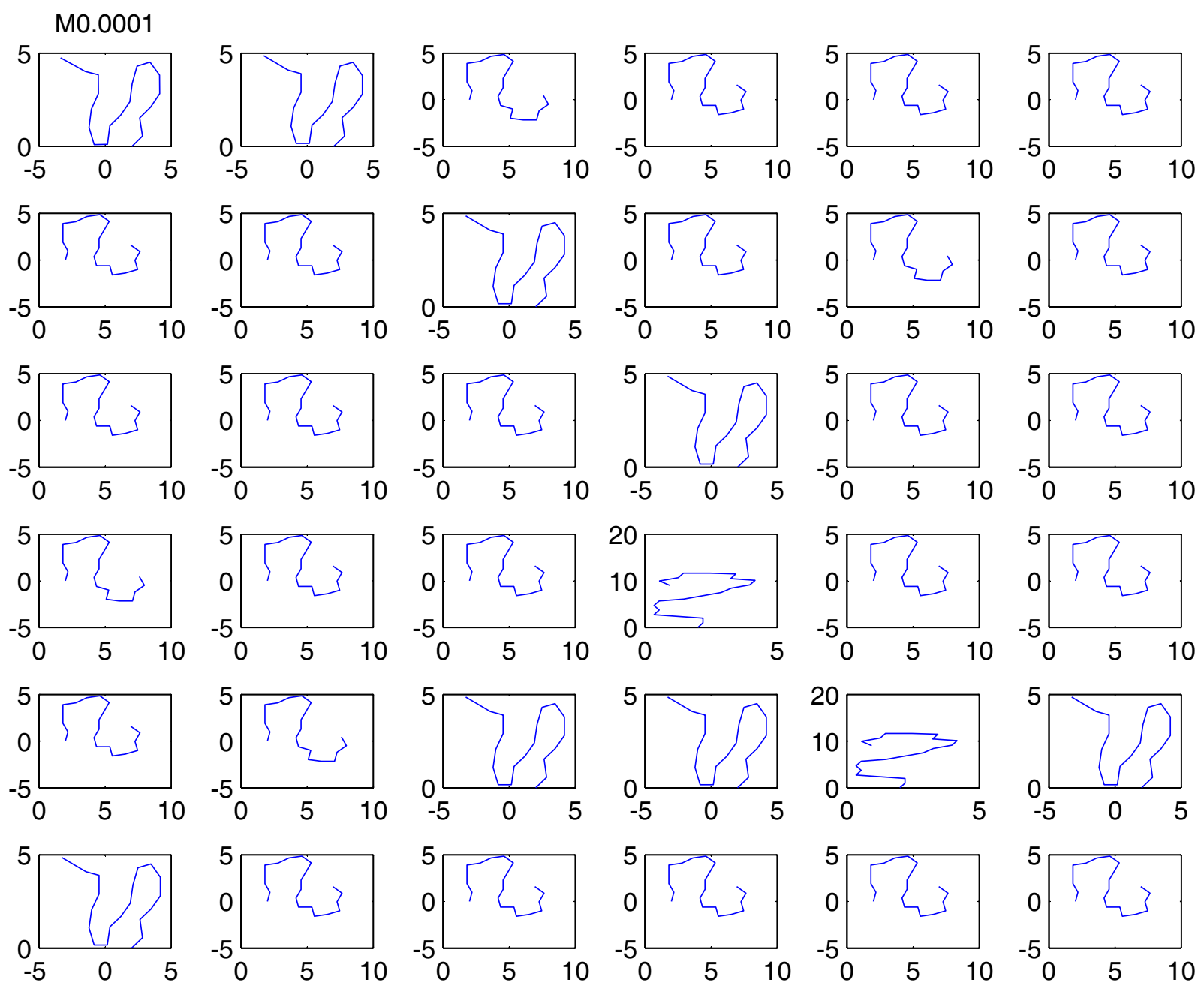

Figure 6a 


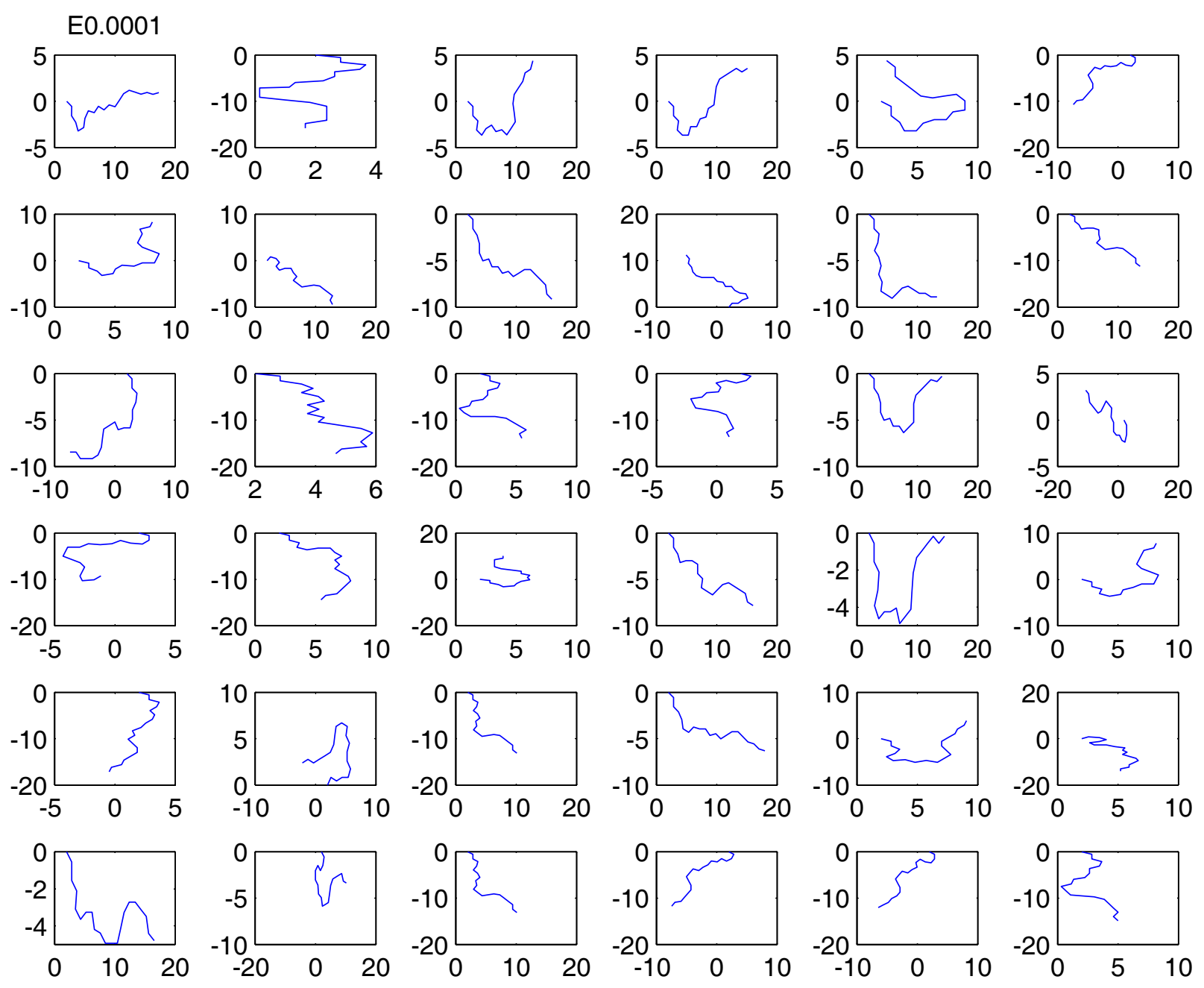

Figure 6b 


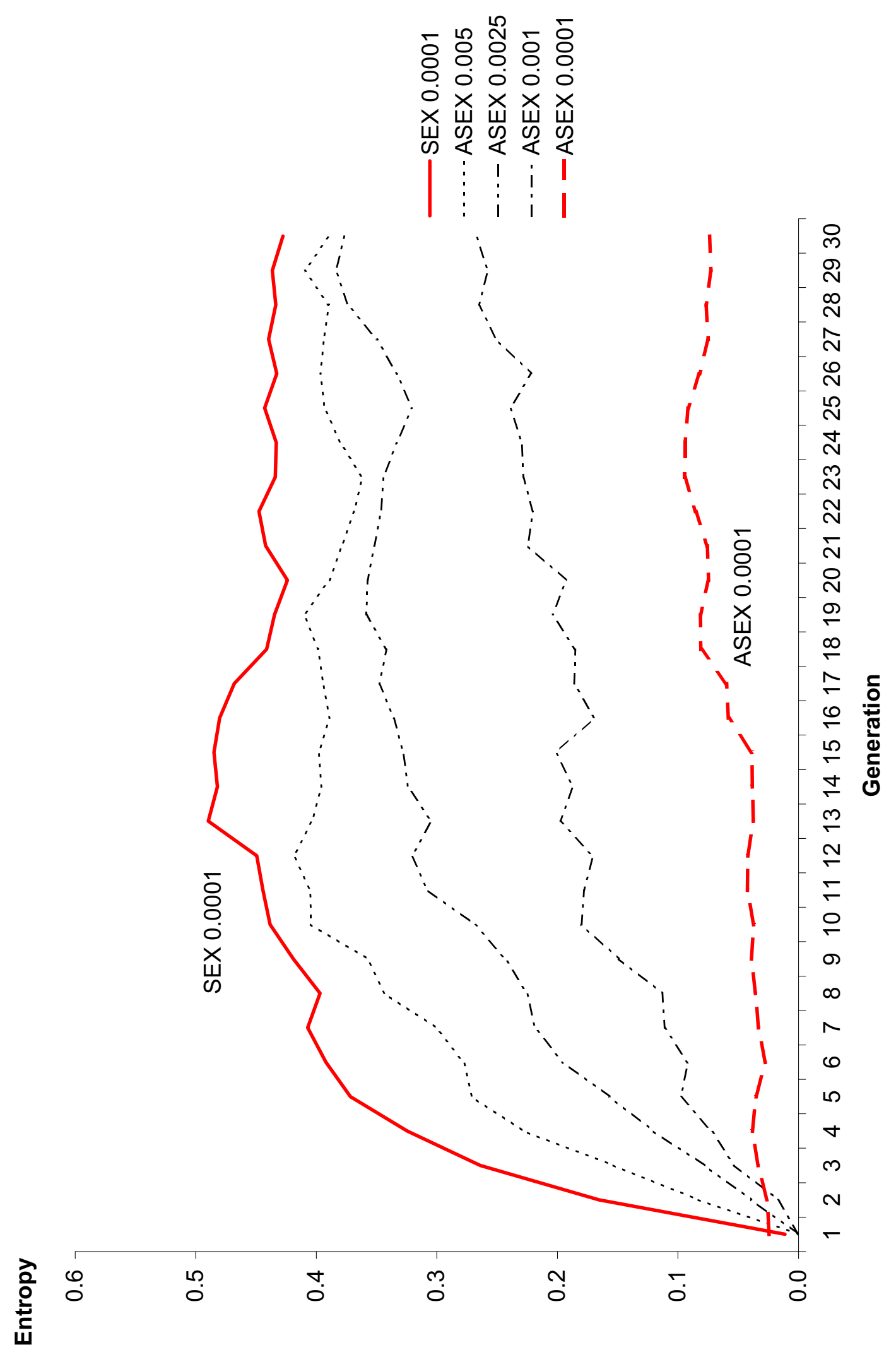

